\title{
Comunicación y política en el cruce de las perspectivas de Jesús Martín-Barbero y de Ernesto Laclau
}

\author{
Ana Lucía Magrini * \\ María Virginia Quiroga **
}

\begin{abstract}
Resumen
El presente artículo exhibe una propuesta de articulación entre la teoría de las mediaciones de Jesús Martín-Barbero y la teoría del discurso político de Ernesto Laclau. El supuesto que guía nuestro análisis sostiene la pertinencia y la relevancia de construir perspectivas de cruce interdisciplinar entre comunicación y política como vía para pensar la pregunta por lo popular y las identidades políticas en América Latina.

Para ello se desarrollará, primero, una aproximación a las propuestas teóricas de Martín-Barbero y de Laclau; en segundo lugar, se presentará un abordaje de las nociones de lo popular y el populismo que se des-
\end{abstract}

* Profesora adjunta de Historia del Pensamiento Político en la Facultad de Ciencia Política y Relaciones Internacionales de la Universidad Católica de Córdoba.

** Docente e investigadora en las carreras de Periodismo (UNSL) y Trabajo Social (UNRC).

Código de referato: SP.221.XLI/17

http://dx.doi.org/10.22529/sp.2017.41.03

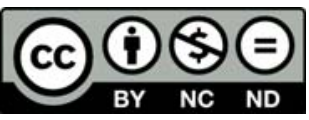

STUDIA POLITICAE sq Número 41 otoño 2017

Publicada por la Facultad de Ciencia Política y Relaciones Internacionales, de la Universidad Católica de Córdoba, Córdoba, República Argentina. 
prenden de las perspectivas de ambos autores. Finalmente, se reflexionará sobre las implicancias teóricas que dichas nociones conllevan para analizar la constitución y redefinición de las identidades políticas en nuestra región.

Palabras clave: Comunicación - Política - Identidades Políticas - América latina

\begin{abstract}
This article shows an articulation proposal between the mediations' theory of Jesús Martín-Barbero and the political discourse theory of Ernesto Laclau. The assumption that guides the analysis reaffirms the pertinence and relevance of building interdisciplinary perspectives between communication and politics, as a way to think about complex issues such as "the popular" and political identities in Latin America.

In the first place, we will try to make an approach to both perspectives. Then, we focus on their notions of popular and populism. Finally, the article reflects on the theoretical implications that such notions carry to analyze the constitution and redefinition of political identities in our region.
\end{abstract}

Key words: Communication - Politics - Political Identities - Latin America

\title{
Introducción
}

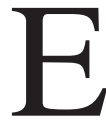

N este artículo, proponemos abordar los posibles puntos de diálogo, especificidades y distanciamientos entre perspectivas teóricas en las cuales la comunicación y la política se cruzan y se yuxtaponen sin instrumentalizarse: ${ }^{1}$ la teoría de las mediaciones de Jesús Martín-Barbero y la teoría del discurso político de Ernesto Laclau. Mientras que la primera ha permanecido inscripta especialmente en el campo de los estudios de la comunicación, la segunda ha predominado en los análisis de la ciencia política, aunque ha establecido vínculos con la filosofía del lenguaje de Wittgenstein, la teoría de la deconstrucción de Derrida y el psicoanálisis lacaniano. No obstante, tanto la teoría de las mediaciones como la teoría del discurso político contribuyeron a proporcionar enfoques novedosos sobre una cuestión que comenzó a cobrar centralidad en América Latina hacia los años ochenta, la problematización de lo popular y la pregunta por las identidades políticas.

1 Esto es, sin reducir la comunicación a una mera estrategia de difusión de información y sin limitar la política a una maquinaria de organización del poder. 
Pero ¿por qué traer a colación la mirada de Jesús Martín-Barbero y la de Ernesto Laclau (y no otras) para abordar las intersecciones entre comunicación y política y, específicamente, para pensar lo popular y las identidades políticas en América Latina?

En primera instancia, porque dichos autores contribuyeron a una teorización sobre América Latina que se inscribe dentro de los estudios postmarxistas. Ambos enfoques forman parte de un proceso de recepción y problematización de los postulados de Antonio Gramsci y constituyen reflexiones en las que los conceptos de discurso, poder, hegemonía y heterogeneidad resultan casi indisociables para pensar ejes problemáticos (lo popular, el populismo y las identidades políticas) que demandan la intervención de análisis políticos y comunicativos. En adición a ello, los autores mencionados desarrollaron nociones no instrumentales de discurso y conceptualizaciones de discursividad no restringidas a la dimensión verbal, en las cuales las relaciones de poder y los procesos de significación fueron concebidos como dimensiones constitutivas de lo social, de lo político y de lo cultural.

Hacia 1978, Martín-Barbero publicó Comunicación masiva, discurso y poder, trabajo en el que el filósofo e investigador colombo-español analizó críticamente los supuestos fundantes de las teorías informacionales, el instrumentalismo funcionalista, el funcionalismo de corte marxista e incluso la complicidad de "la lingüística estructural (...) con la teoría cibernética de la comunicación” (1978, p. 36). Nueve años más tarde apareció una de las obras más referenciadas del autor: De los medios a las mediaciones. Comunicación, cultura y hegemonía. El libro se produjo en un peculiar contexto de debate que puso en cuestión supuestos de base de la comunicación social y de las ciencias sociales. El primer efecto que produjo la intervención de Martín-Barbero sobre los debates comunicacionales fue la tematización de la necesidad de pensar América Latina desde marcos teóricos que habilitaran investigar problemas comunicativos, culturales, políticos y sociales construidos en el contexto latinoamericano.

Por su parte, Ernesto Laclau publicó en 1977 Politics and Ideology in Marxist Theory ${ }^{2}$, donde propuso una teoría del populismo que intentaba tomar distancia de las interpretaciones producidas desde la teoría de la modernización y desde el marxismo. Frente a ello, Laclau sostuvo que el carácter de clase de una ideología debía buscarse, no en la presencia de determinados contenidos en un discurso, sino en el principio de articulación que los unificaba. En Hegemonía y estrategia socialista (1987

2 Entre las que se destacan: LACLAU (1996, 2000, 2002). 
[1985]), Ernesto Laclau y Chantal Mouffe profundizaron aquella empresa de lectura crítico-deconstructiva de la tradición marxista, desmantelando los supuestos esencialistas del marxismo clásico e incluso de su "último reducto esencialista” (la teoría gramsciana). Los autores propusieron una mirada de lo político que no negaba el antagonismo y los procesos de significación de lo social sino que por el contrario los consideraba como elementos constitutivos de toda acción social y política. Posteriormente, y luego de numerosas obras, ${ }^{3}$ en 2005 Ernesto Laclau publicó La razón populista, trabajo que contiene más explícitamente la propuesta teórica del autor respecto al concepto de populismo. Vale recordar que esta última etapa del desarrollo de la teoría laclausiana se desplegó en el marco de un debate por distinguir "la política" de "lo político". 4

En segundo lugar, resulta pertinente recuperar y articular las reflexiones de Martín-Barbero y las de Laclau en tanto aportaron elementos clave para un descentramiento relevante que cobró auge en los 80 y que aún sigue repensándose: abordar lo comunicativo y lo político en tanto procesos. En este sentido, Martín-Barbero construyó un mapa teórico en el que los medios de comunicación perdieron su lugar totalizador; ello implicó una ruptura con la teoría informacional y advirtió la necesidad de dejar de pensar en los medios masivos como meros artefactos tecnológicos para abordar el problema de la comunicación como un proceso necesariamente mediado. Mientras tanto, Ernesto Laclau propuso pasar de una mirada politológica centrada en las dinámicas ónticas de la acción política convencional (la política) a una perspectiva que aborde la pregunta por los modos de producción de sentidos sobre lo político. Desde este prisma, se buscó trascender los estudios exclusivamente preocupados por la enunciación de los líderes para indagar en los procesos (históricos y discursivos)

3 Mientras que la política involucra una serie de prácticas ónticas de la acción política convencional y gubernamental (sistema de partidos, acciones de gobierno, competencia electoral, creación de legislación, entre otras cuestiones), lo político designa una dimensión ontológica que refiere a las formas y modos en que se producen sentidos sobre lo social como tal (MARCHART, 2009).

4 El autor identifica cuatro formas posibles de mediación: regímenes de institucionalidad [lectura que convierte a la comunicación en cuestión de medios masivos en cuanto producción de discursos públicos hegemónicos, los cuales median entre las lógicas de producción (LP) y las matrices culturales (MC)], formas de socialidad [estas refieren a la dimensión de la cotidianidad]; la comunicación como socialidad se dirime en cuestión de fines, y media entre las matrices culturales (MC) y las competencias de recepción (CR). Ritualidades [mediadoras de las relaciones entre las competencias de recepción (CR) y los formatos industriales (FI)]; y tecnicidades [que median entre las lógicas de producción (LP) y los formatos industriales (FI)]. 
de construcción de fronteras políticas, de antagonismos y de identidades populares.

Consideramos que una propuesta de cruce entre dichos enfoques teóricos resulta pertinente porque ambos impulsaron formas para leer lo político y lo comunicativo "desde arriba”, esto es, desde la política y la comunicación que pasa por lo hegemónico; pero también “desde abajo”, es decir, desde la recepción, la comunicación y la política que pasa por lo cotidiano y por los procesos de identificación populares.

Finalmente, vale señalar que en este trabajo se recurrió a una metodología cualitativa que combina aportes de las teorías de la comunicación y la teoría política. En el recorrido se intentó seguir una lógica inductiva, ya que a partir del análisis de las propuestas de Martín-Barbero y de Laclau se plantearon interrogantes más generales sobre la constitución de las identidades políticas en América Latina. Se argumenta, en consecuencia, que la pregunta por lo popular y las identidades políticas es un tema clave en la intersección entre comunicación y política.

\section{Aproximaciones a la perspectiva de Jesús Martín-Barbero: comunicación, mediaciones y discursividad}

Hacia finales de los años setenta, Martín-Barbero advertía una suerte de homogeneidad subyacente en las teorías comunicacionales: éstas recurrían a una noción de lenguaje como un instrumento de la comunicación-transmisión. Frente a este supuesto, propuso pensar la comunicación "como un proceso desgarrado por las contradicciones y el conflicto de intereses antagónicos” (1978, p. 26). En este sentido, la perspectiva de Martín-Barbero parte de una lectura crítica del marco conceptual de las teorías del discurso. El filósofo y comunicador identificaba en el modelo del signo y en el método lingüístico-estructuralista, así como en los enfoques marxistas de carácter universalizante, tentaciones formalistas y tendencias a mantener supuestos propios del funcionalismo. Observaba la dificultad de establecer relaciones entre textos y contextos histórico-sociales, la predisposición a configurar un concepto de sentido unívoco y conceptualizaciones de los sujetos como entidades vinculadas desde un solo tipo de relación (privilegio de las relaciones de clase, por ejemplo). De allí la necesidad de "sacar" a la comunicación del nivel superestructural en el que los estudios marxistas puros y los enfoques lingüísticos y estructuralistas clásicos la habían colocado.

La noción de discurso propuesta por Martín-Barbero cuestiona la reducción del lenguaje a lo lingüístico, ya que es desde "los bordes de lo lin- 
güístico (...) por donde es minada la paz de la estructura, descentrándola hacia un 'exterior' en el que se 'libera' su poder” (1978, p. 122-123). Su perspectiva recupera la dimensión ideológica del discurso y la concibe, ya no como un reflejo de la superestructura, sino como una cuestión constitutiva y constituida en el mismo proceso de producción del discurso. Su mirada de lo discursivo parte de una noción de subjetividad en la que los sujetos "no se definen por algún tipo de intencionalidad sino por el 'lugar' que ocupan en el espacio social y por la forma como inscriben su presencia en el discurso" (1978, p. 121). Desde este punto de vista propone mostrar "la malla de relaciones por las que el Poder actúa, se realiza, no aparte, no por fuera, sino a través de las relaciones sociales, económicas, políticas, culturales, sexuales” (1978, p. 45).

Se traza allí un nexo con las reflexiones de Michel Foucault en El orden del discurso. En esa vía, Martín-Barbero entiende al discurso desde una noción no divorciada de la acción social y desde un específico concepto de práctica: “discurso como práctica discursiva, no como algo que está ahí y que después hay que ver cómo se lo relaciona con el modo de producción, sino como parte integrante, constitutiva de él” (1978, p. 46). Ello constituye el eje del desplazamiento hacia un concepto de comunicación como discurso del poder.

El problema de la comunicación deja de ser un problema de contenidos significativos ya que esa perspectiva, en su reducción del discurso a relaciones de significación, de lengua (...), descarta su hacer, su movimiento, su trabajo y por tanto su inserción en él (...) del sujeto histórico y pulsional (1978, p. 46).

Las reflexiones anteriores constituyen la base para el abordaje de lo discursivo como un proceso mediado, cuestión que se explicita en $D e$ los medios a las mediaciones... En dicha obra, el concepto de mediación se encuentra íntimamente vinculado con la teoría de la hegemonía de Gramcsi, de allí que la noción de dominación va a ser puesta en cuestión a partir de la pregunta por los procesos de recepción. Adicionalmente, la idea de dominación se complejiza al identificar relaciones que median entre quienes la ejercen y quienes son "objeto" de ella (los dominados).

La tesis sobre las mediaciones podría sintetizarse a partir de un esquema configurado por dos ejes: uno histórico-diacrónico, compuesto por matrices culturales (MC) y formatos industriales (FI), y otro sincrónico, constituido por lógicas de producción (LP) y competencias de recepción o consumo (CR). Estas relaciones diacrónicas y sincrónicas remiten a los procesos históricos, políticos, económicos y culturales que hacen 
comprensibles los cambios en las articulaciones de los grupos sociales y los discursos públicos. En última instancia, se trata de hacer visibles los desplazamientos en las formas hegemónicas de comunicación pública y colectiva.

Más allá de las formas posibles en que las mediaciones tienen lugar, ${ }^{5}$ interesa destacar que Martín-Barbero construye un mapa de procesos comunicativos en el que los medios pierden su centralidad como elemento esencial de la comunicación, ésta comienza a abordarse como un problema que involucra procesos necesariamente mediados.

Lo que busco en este mapa es reconocer que los medios constituyen hoy espacios claves de condensación e intersección de múltiples redes de poder y de producción cultural, pero alertar al mismo tiempo contra el pensamiento único que legitima la idea de que la tecnología es hoy el "gran mediador" entre los pueblos y el mundo, cuando lo que la tecnología media hoy más intensa y aceleradamente es la transformación de la sociedad en mercado (...) (Martín-Barbero, 2003 [1987], p. 21).

En suma, la teoría de las mediaciones permite pensar las relaciones entre comunicación y política desde una perspectiva en la cual la comunicación se define como proceso mediado y como discurso atravesado por relaciones de poder. Ello implica analizar los componentes simbólicos e imaginarios de los procesos de formación del poder como dimensiones constitutivas de todo proceso de producción social de sentidos. Por otra parte, la crítica a las visiones cerradas de estructura y a las miradas "lingüocéntricas" del discurso, contribuyen a la apertura de la comunicación hacia la pregunta por el sujeto y los procesos de recepción. Parafraseando a MartínBarbero, la comunicación como mediación se preocupa más que por los medios en sí, por qué cosas hace la gente con ellos.

\section{Aproximaciones a la perspectiva de Ernesto Laclau: discurso y articulaciones políticas}

La teoría del discurso político de Ernesto Laclau también supone una lectura crítica de la lingüística clásica, sin embargo su noción de discurso no intenta sortear el modelo del signo sino recuperarlo despojándolo de sus for-

\footnotetext{
5 Interrogar las tensiones entre mediación y articulación requeriría reconstruir los debates entre hermenéutica y postestructuralismo, cuestión que excede los objetivos de este trabajo. Para una aproximación a este tipo de análisis véase: VerGALITO, 2008.
} 
malismos y supuestos universalizantes. Desde la perspectiva laclausiana todo discurso comprende instancias del habla, "aquello que los actores dicen”, y las prácticas sociales, “aquello que los actores hacen”. Esta noción material de discurso supuso un replanteo de la propuesta del lingüista suizo Ferdinand de Saussure. De acuerdo con el esquema saussureano, existe una estructura abstracta que determina las manifestaciones concretas de la lengua. El significado de las palabras no es esencial sino relacional, por lo tanto el signo lingüístico es arbitrario y el lenguaje es un sistema de diferencias en el cual los significantes solo tienen sentido por su opuesto y no dependen de la realidad que están expresando, sino de otros términos con los que están en relación.

Ahora bien, desde la mirada de Laclau, el estructuralismo saussureano había dado como resultado un enfoque "lingüocéntrico" que se había ocupado de las reglas internas del sistema de la lengua apartándose cada vez más del estudio de la realidad social. Ello había llevado a la configuración de un método de análisis del discurso centrado en la identificación de las unidades discretas entre significantes, así como en el establecimiento de sus relaciones y jerarquías, pero en un contexto básicamente textual. Conforme con Laclau, el problema del enfoque lingüístico-estructural radicaba en que partía de un supuesto ontológico en el que la contingencia, la exterioridad y el antagonismo no resultaban posibles. En este punto reside una de las particularidades de la propuesta laclausiana al señalar que el lenguaje no es un sistema cerrado de diferencias sino abierto; ya que para afirmar que un sistema lingüístico es cerrado — supuesto básico de la lingüística de Saussure-, deberíamos determinar primero una frontera y para poder identificar dicha frontera necesariamente debería presentarse algo que se encuentre fuera de ésta, un elemento excluido (Laclau, 2002).

En definitiva, el esquema de Laclau comparte con el de Saussure que los elementos presentes en el discurso se definen relacional y arbitrariamente por oposición a otros, pero se diferencia en que el discurso se encuentra abierto y los significantes que lo constituyen adquieren unidad relativa a partir de un elemento que se encuentra excluido de aquellas relaciones internas. Dicho elemento excluido es clave para comprender la lógica del antagonismo y de la hegemonía en la teoría del discurso político laclausiana. Lo excluido representa, de un lado, lo externo, lo Otro antagónico al que los elementos internos del discurso se oponen, y de otro lado, aquello que al mismo tiempo resulta constitutivo del propio discurso. En este sentido, la exterioridad representa una condición de posibilidad para producir unidades de sentido internas a todo discurso, aunque estas se verán siempre amenazadas y/o desestabilizadas por aquello que se encuentra por fuera de las propias fronteras. 
¿Cómo se vincula esta concepción de discurso con el abordaje de lo político? En principio vale señalar que ambas cuestiones se encuentran mutuamente imbricadas: la especificidad de lo político se define desde lo antagónico y la especificidad de lo discursivo implica lucha por imposición de sentidos. De allí que todo acontecimiento político es entendido como un hecho polémico, atravesado por relaciones de poder y constantemente disputado por otros discursos. Las relaciones de oposición y de arbitrariedad del modelo del signo son retomadas por Laclau desde una lectura material y no esencialista del discurso. Desde nuestro punto de vista, esta noción de discurso resulta pertinente para abordar lo político, lo histórico y lo comunicativo al incluir la dimensión de las prácticas sociales dentro del campo del discurso. Así, discurso implica procesos de lucha por imposición de sentidos desde un supuesto central: el carácter constitutivo (no divorciado) de lo político, lo social y lo discursivo.

En suma, es posible encontrar algunos puntos de contacto entre los abordajes sobre lo discursivo en las reflexiones de Jesús Martín-Barbero y de Ernesto Laclau: (a) ambos autores presuponen una noción de discurso que nos permite problematizar los formalismos de la lingüística estructural y los determinismos del marxismo clásico; (b) parten de considerar que el lenguaje y lo discursivo no son elementos divorciados del mundo social, sino constitutivos de este, lo que nos permite abordar no solo los componentes significantes de la producción social de sentido, sino también el modo en que éste se construye desde las prácticas sociales y la acción política; (c) ello tiene una consecuencia de orden ontológico relevante: el carácter necesariamente mediado (Martín-Barbero) y articulado (Laclau) de lo comunicativo y de lo político. Más allá de las posibles distinciones y tensiones entre articulación y mediación, ${ }^{6}$ ambos conceptos suponen procesos en los que se asume la imposibilidad de aprehender de manera directa los objetos o fenómenos, ya que estos se encuentran constantemente atravesados por articulaciones políticas y por mediaciones comunicativas.

\section{Populismo e identidades políticas: algunos puntos de diálogo entre las perspectivas de Jesús Martín-Barbero y de Ernesto Laclau}

Parafraseando a Umberto Eco (1968) la noción de populismo se debate en América Latina entre discursos "apocalípticos” y discursos "integrados".

6 Véase: MARTín-BARBERo, 2009. 
Mientras que los primeros entienden al populismo como sinónimo de demagogia y de clientelismo político, los segundos reivindican el fenómeno como un proceso capaz de expandir la democracia. Dada la polisemia del concepto y las múltiples polémicas que ha suscitado, resulta oportuno interrogarnos sobre las contribuciones que las perspectivas analizadas en los apartados anteriores pueden realizar a la hora de (re)pensar la problemática del populismo y lo popular en nuestra región.

Jesús Martín-Barbero y Ernesto Laclau tomaron distancia de la búsqueda por identificar el "contenido esencial" de estos tópicos. Ello implicaba restar centralidad a las explicaciones que remitían a contextos históricos específicos, criterios del deber ser, de la política y/o procesos desviados del curso "normal" de la evolución histórica. Siguiendo esas líneas de abordaje, se llegaba a una definición de populismo asociada a fenómenos peyorativos, como el caudillismo, el autoritarismo, el clientelismo político, entre otros. En este sentido, lo popular recibía también connotaciones negativas herederas de una concepción liberal que lo asociaba a lo vulgar, lo irracional y lo inmaduro.

Ahora bien, si el populismo y lo popular constituyen conceptos que se debaten entre enfoques diversos, la categoría de identidad no carece de tal contingencia. En nuestra región, asistimos a un uso cada vez más frecuente de este concepto y a la presencia de sentidos un tanto ambiguos para definirla. Identidad de clase, de los trabajadores, de las mujeres, identidades étnicas, modos identificatorios, son algunas de las acepciones que solemos encontrar en el vocabulario académico sobre el tema. En relación a las perspectivas sobre la identidad es posible advertir el predominio de enfoques sustancialistas, los cuales buscan dar cuenta de la esencia-fundamento sobre la que se define o construye una identidad determinada. Algunas consecuencias que comúnmente se derivan de estos estudios refieren a que: (1) suponen que la identidad o más recientemente las identidades se constituyen a partir de una suerte de cultura homogénea, en algunos casos ésta también es concebida en términos estáticos; (2) presumen que la identidad o las identidades, culturales o políticas, se encuentran sustentadas por una serie de principios que las autodefinen (ejemplo de ello es el caso de las identidades nacionales, que suelen definirse desde principios tradicionales como la historia común, la música, la lengua, el territorio, entre otros); (3) generalmente no se establece un análisis capaz de dar cuenta de las dimensiones conflictivas y simbólicas de los procesos de constitución y re-constitución identitarios.

Frente a ello, los modos en que las perspectivas de Martín-Barbero y de Laclau problematizaron lo popular y el populismo conllevan implicancias 
pertinentes para un abordaje no esencialista ni sustancialista de las identidades políticas y populares (Magrini, 2010). En principio, la teoría de las mediaciones de Jesús Martín-Barbero parte de una distinción fundamental entre lo popular y lo masivo. Lo popular "hace posible la expresión de las aspiraciones y expectativas colectivas producidas por y desde los grupos sociales de base. Tanto mayoritarios como minoritarios, tanto a nivel patente como latente" (2002, p. 118). Lo masivo se presenta como una negación de lo popular y remite a una cultura producida para su masificación y control, un tipo de cultura con tendencia a negar las diferencias. No obstante, la lectura de Martín-Babero complejiza la mutua oposición entre ambos términos al advertir que lo masivo se configura como una mediación de lo popular en la cual las expectativas y los sistemas de valoración, "los gustos populares", son moldeados interpretados o leídos desde lo masivo. "Lo masivo es entonces la imagen que la burguesía se hace de las masas, o mejor la imagen de sí mismas que estas deben interiorizar para que cotidianamente sea legitimada la dominación que aquella ejerce" (2002, p. 119). El filósofo y especialista en comunicación propone entonces pensar lo popular desde su imbricación masiva, ello implica no hacer de lo masivo un mero sinónimo de alienación y manipulación, ya que la masividad no remite a un mecanismo aislable de lo social, sino que se encuentra en su propia lógica, en el sistema educativo, en las formas de representación, en las prácticas religiosas, en los modos de consumo y hasta en los usos del espacio (2003, p. 319). Desde este punto de vista, lo popular involucra diversidad y heterogeneidad, muchas veces de carácter ambiguo y conflictivo.

Si pensamos el concepto de populismo desde estas reflexiones podríamos advertir que ha sido la idea de masas como mayorías irracionales y fácilmente manipulables la que ha predominado en las nociones peyorativas del populismo, en las que masa y pueblo se identifican mutuamente y se consideran como la base social de formas políticas autoritarias ("populistas").

Desde el espacio de la política oficial, a derecha y a izquierda, tanto las masas como lo masivo serán mirados con recelo. La derecha con una posición a la defensiva: las masas ponen en peligro acendrados privilegios sociales, y lo masivo disuelve sagradas demarcaciones culturales. La izquierda ve en las masas un peso muerto, un proletariado sin conciencia ni clase, ni vocación de lucha, y en lo masivo un hecho cultural que no cuadra en su esquema, que desafía e incómoda su razón ilustrada. Solo para los populistas la presencia de la masa urbana pareció implicar un hecho político nuevo ... (Martín-Barbero, 2003, p. 218). 
Frente a la renovada centralidad de esta temática en la década de los 80, Martín-Barbero señala que el populismo en América Latina se explicaría por la compleja tensión entre el proceso de masificación y la emergencia de lo popular. De acuerdo al autor, en este contexto resulta central la intervención de Ernesto Laclau, quien para esa época identificó una serie de conflictos que no coinciden totalmente con las relaciones de clase y de producción, sino que remiten a "una contradicción diferente y específica que se sitúa en el plano de las formaciones sociales y 'que opone antagónicamente el pueblo al bloque en el poder'” (Laclau, 1978, p.122, en Martín-Barbero, 2003, p. 222).

A partir de estas consideraciones sobre lo masivo, lo popular y el populismo, el autor colombo-español plantea la necesidad de construir agendas de investigación para América latina ${ }^{7}$ que incluyan estudios sobre el paradójico proceso de vinculación entre lo masivo y lo popular. Ello demanda para Martín-Barbero una serie de transformaciones en el qué investigar (el objeto de la comunicación propiamente dicho) y en el cómo investigar (estrategias metodológicas que exigen una reflexión respecto a sus implicancias, no solo técnicas, sino respecto a los supuestos de carácter ideológico que las motivan). Y es aquí donde el autor propone una perspectiva que recupera el enfoque histórico-discursivo. Los diálogos entre comunicación e historia ya no remitirían a la comúnmente desarrollada historia de los medios, sino a una historia de los procesos culturales, concebidos como articulaciones entre prácticas comunicativas, identidades políticas y movimientos sociales. Ello sustancialmente comprende "ubicar la comunicación en el espacio de las mediaciones en las que los procesos económicos dejan de ser un exterior de los procesos simbólicos y éstos a su vez aparecen como constitutivos y no solo expresivos del sentido social” (2002, p. 128). Este viraje otorga protagonismo a la pregunta por cómo las identidades populares se han hecho visibles en el espacio público en América latina y a través de qué prácticas políticas y comunicativas han construido sus luchas.

Bajo este lente analítico las identidades populares son entendidas como procesos de identificación colectivos, heterogéneos, dinámicos, relacionales, contingentes y atravesados por relaciones de poder. La teoría de las mediaciones sostiene que las identidades se configuran en medio de relaciones de tensión y lucha en el terreno simbólico, ya que es allí donde "se articulan las interpelaciones desde las que se constituyen los sujetos, las identidades colectivas” (Martín-Barbero, 2003, p. 288).

\footnotetext{
7 Véase también: MARTín-BARBERO, 1984.
} 
En este sentido, la propuesta de Martín-Barbero se encuentra en sintonía con la perspectiva laclausiana sobre el populismo y las identidades populares. Para Ernesto Laclau el populismo es fundamentalmente un tipo de discurso que "construye a los individuos como sujetos desde formas de interpelación bajo las cuales los sectores dominados no se identifican a sí mismos en tanto clase social, sino como 'lo otro', 'lo opuesto' al bloque de poder dominante, como los de abajo” (1980 [1977], p. 220).

El efecto teórico y político de su intervención fue la constitución de un concepto no esencialista de populismo, esto quiere decir que no es posible identificar un contenido ideal o normativo previo a la constitución del fenómeno. Éste carece de especificidad histórica (no remite a un período determinado del desarrollo capitalista) y de especificidad geográfica (no es propio de los países del Tercer Mundo). En última instancia, el populismo, bajo el lente de Laclau es contingente, flexible, no cerrado y constantemente disputado.

...el populismo no tiene ninguna unidad referencial porque no está atribuido a un fenómeno delimitable, sino a una lógica social cuyos efectos atraviesan una variedad de fenómenos. El populismo es, simplemente, un modo de construir lo político (Laclau, 2005, p. 11).

De acuerdo con las teorizaciones más recientes de Laclau, el discurso populista constituye una forma de articulación de lo político que actúa según la lógica de la equivalencia. Ésta alude al proceso por el cual se construyen solidaridades entre determinados discursos a partir de la negación de la satisfacción de distintas demandas. Las mismas demandas son equivalentes en relación a aquello que las niega, la institucionalidad que no les da lugar; por ello se dice que sus lazos equivalenciales son de carácter negativo. De este modo, a través del surgimiento de una cadena equivalencial de demandas insatisfechas, se construye una frontera interna que dicotomiza el espacio social: por un lado, el campo de la institucionalidad excluyente, el lugar de los poderosos; por el otro, el lugar de los excluidos, los que no obtienen respuesta, y que Laclau resume en la idea de "los de abajo" [underdogs].

Hasta aquí estaríamos frente a la presencia de dos de las precondiciones del populismo: una frontera interna antagónica y una articulación equivalencial de demandas. El tercer requisito, para Laclau, refiere a la consolidación de la cadena equivalencial mediante la construcción de una identidad popular, que es cualitativamente algo más que la suma de los lazos equivalenciales. La creación del pueblo — distinto a la masa — supone la existencia de una particularidad con pretensiones hegemonistas; una parcialidad que, en nombre del daño que la comunidad le ha provocado, aspira a repre- 
sentar el todo comunitario. De allí la idea de "una plebs que reclama ser el populus legítimo de la comunidad” (2005, p. 108).

¿Cómo se articula entonces esta conceptualización del populismo con las identidades políticas? Retomando los aportes de Jacques Lacan sobre los modos identificatorios y sobre la noción de falta, Laclau sostuvo que toda identidad colectiva es relacional, se construye por oposición a un otro y se vincula con una concepción de subjetividad escindida o fallada, es decir, que el sujeto se constituye como sujeto de una falta. De allí que la teoría del discurso político supone el carácter precario, dinámico y heterogéneo de las identidades políticas.

En definitiva, desde la perspectiva laclausiana las identidades políticas se definirían a partir de los siguientes supuestos: (a) se trata de modos identificatorios construidos en el mismo proceso de configuración hegemónica (esto quiere decir que no son esenciales ni predeterminadas); (b) para la formación de las identidades resulta clave la lógica del antagonismo, perspectiva desde la cual una identificación se construye por oposición a un otro, exterior y al mismo tiempo constitutivo; (c) toda identidad está constitutivamente dividida entre su reivindicación particular y un elemento que entra en articulación con otros a partir de la oposición a un enemigo común.

Estudios posteriores, en la órbita de la teoría del discurso político, han operacionalizado el concepto de identidad política para analizar casos históricos y contemporáneos de la experiencia latinoamericana (Aboy Carlés, 2001; Quiroga, 2016). En este sentido, se han identificado dimensiones y subdimensiones para facilitar el análisis empírico, buscando tomar distancia de un entendimiento formal y rígido de las identidades. Se trata de aportes que intentan dejar en evidencia el carácter disputado y, por tanto, re-definible de cualquier fenómeno/proceso social, comunicativo y político.

\section{Consideraciones finales}

Lo popular, el populismo y las identidades políticas constituyen tópicos de investigación que requieren del trazado de puentes teóricos y analíticos entre comunicación y política. En este sentido, la comunicación se presenta como mediación y la política como articulación, en la búsqueda por aprehender procesos y fenómenos constitutivamente contingentes, históricos, flexibles y disputados. De ese modo, tanto la teoría de las mediaciones de Jesús Martín-Barbero como la teoría del discurso político de Ernesto Laclau parten del supuesto de que el lenguaje y lo discursivo no son elemen- 
tos divorciados del mundo social, sino constitutivos de éste. Dicha tesis habilita un abordaje del populismo y lo popular desde las prácticas, las articulaciones y las mediaciones político-comunicativas. Al mismo tiempo, conlleva una consecuencia de orden ontológico relevante para los estudios interdisciplinarios: el carácter necesariamente mediado de lo comunicativo y el carácter necesariamente articulado de lo político. De allí que los fenómenos populistas se encontrarían atravesados por articulaciones y tensiones entre la lógica de la diferencia y la equivalencia (Laclau, 2005) y por mediaciones de orden institucional, social, cultural, rituales y tecnológicas (Martín-Barbero, 2003).

Como advertimos, el populismo y las identidades políticas remiten a conceptos disputados, polémicos y polisémicos que se construyen en el marco de una serie de debates que resultaron especialmente prolíficos en América latina hacia los años ochenta. En este marco, Barbero y Laclau propusieron miradas no esencialistas del populismo y no sustancialistas de las identidades políticas y populares. Sus reflexiones problematizaron además los fundamentos de lo político y lo comunicativo, deconstruyeron los supuestos esencialistas de las teorías de la comunicación y de la teoría política, y propusieron abordajes teóricos que se posicionaron en los márgenes entre estas disciplinas.

En suma, un enfoque discursivo y político-comunicativo para pensar lo popular, el populismo y las identidades políticas en América latina implicaría interpretar nuestros objetos de estudio "desde arriba y desde abajo", abordando lo comunicativo como un proceso que se configura desde la enunciación, las mediaciones y la recepción; y lo político desde la hegemonía, el antagonismo, las articulaciones entre las identidades políticas, las resistencias e incluso las complicidades de la memoria popular con lo masivo y con lo hegemónico.

要

\section{Referencias bibliográficas}

Aboy CARLÉs, G. (2001). Las dos fronteras de la democracia argentina. La redefinición de las identidades políticas de Alfonsín a Menem. Rosario: Homo Sapiens.

ECO, U. (1968). Apocalípticos e integrados ante la cultura de masas. Barcelona: Palabra en el Tiempo.

Laclau, E. y Mouffe, C. (1987 [1985]). Hegemonía y estrategia socialista. Madrid: Siglo XXI.

Laclau, E. (1980 [1977]) Política e ideología en la Teoría Marxista, Capitalismo, Fascismo, Populismo. México: Siglo XXI.

—. (1996). Emancipación y diferencia. Buenos Aires: Ariel. 
LAClAu, E. (2000) Nuevas reflexiones sobre la revolución de nuestro tiempo. Buenos Aires: Ediciones Nueva Visión.

- (2002) Misticismo retórica y política. Buenos Aires: FCE.

(2005) La razón populista. Buenos Aires: FCE.

MARChART, O. (2009). El pensamiento político posfundacional. La diferencia política en Nancy, Lefort, Badiou y Laclau. Buenos Aires: FCE.

MARTín-BARBero, J. (1978). Comunicación masiva, discurso y poder. Quito: Editorial Época.

_ (1984). "De la comunicación a la cultura. Perder el "objeto” para ganar el proceso”. Signo y Pensamiento 5, (3), 17-24.

. (2002). Oficio de cartógrafo. Travesías latinoamericanas de la comunicación en la cultura. Bogotá: FCE.

. (2003 [1987]). De los medios a las mediaciones. Comunicación, cultura y hegemonía. Bogotá: Convenio Andrés Bello.

QuirogA, M. V. (2016). Identidades políticas y movilización social. Un estudio comparado de la CTA en Argentina y el MAS-IPSP en Bolivia. Eduvim: Villa María.

Vergalito, E. (2008). Acción, decisión e identidad política: del postestructuralismo a la hermenéutica. (Tesis de maestría inédita). Facultad de Ciencias Sociales, Universidad de Buenos Aires, Argentina.

Fecha de recepción: 27/08/2016

Fecha de aceptación: 09/12/2016 